\title{
Small Galaxy Groups in the Universe
}

\author{
A. Cavaliere \\ Università di Roma, Italy
}

\begin{abstract}
.
I discuss the steering role played by small groups of galaxies concerning sources and structures on widely different scales: the very compact quasars, and the extended atmospheres of hot intracluster gas. The evolution of such sources out to substantial $z$ is ruled by astrophysical processes taking place in early small groups.
\end{abstract}

\section{Introduction}

My discussion will be based on the properties of small groups (SGs) as defined by local surveys (see Karachentsev et al. 1999, Ramella et al. 1999), and projected to higher $z$ after the standard hierarchical clustering.

This envisages the galaxies as baryonic condensations within heavier halos of cold dark matter (DM), which frame their dynamical evolution while themselves scale up in mass by repeated merging with similar structures. SGs with mass $M \sim 10^{13} M_{\odot}$ began to form at a considerable rate from $z_{G} \approx 2.5$ in most viable cosmologies. Before $z_{G}$ (proto)galaxies are built up by merging of smaller units; around $z_{G}$ the galaxies are assembled into SGs, which later on merge into richer groups and into clusters. The variance in such epochs is wide, and for the net populations in each mass range the final demise is slow.

\section{Quasars and small groups}

On the other hand, the bright quasar (QSO) population culminates at $z_{Q} \approx$ $3 \pm 0.5$, but it falls steeply by a factor $10^{2}$ going to lower redshift (Shaver et al. 1997 , Osmer 1998). Is the near equality $z_{Q} \approx z_{G}$ just a coincidence? Does the sharp QSO fall relate to the gentle demise of the SGs over the same range?

$I$ argue the link to be intrinsic, assuming the QSOs are powered by massive black holes (BHs) that accrete gas from their host galaxies; the luminosity

$$
L \sim \eta c^{2} \Delta m / \Delta t \lesssim 10^{48} \mathrm{erg} \mathrm{s}^{-1}
$$

is obtained when the mass $\Delta m$ is accreted over the time $\Delta t$ with conversion efficiency up to $\eta \sim 10^{-1}$ (Rees 1984). Such huge bolometric powers imply fractions emitted in the visible (canonically some $10^{-1}$ ), IR or X-rays large enough to allow the conditions prevailing in the QSO environment to be detectible from $z \sim 5$.

Earlier than $z_{G}$ such an environment is constituted by single (proto)galaxies (see Steidel et al. 1998) being built up by major, chaotic merging events be- 
tween gas-rich units; the central BHs coalesce and/or accrete rapidly from the abundant quantities of available gas (Haehnelt et al. 1998). In these events the gravitational potential is strongly distorted, while the baryons are clumped and shocked; so the angular momentum $j$ of the gas orbiting at kpc distances in the host is rapidly transferred to the heavier DM substructures. Hence the gas can begin an inward course to eventually settle onto the accretion disk at pc and smaller scales.

Thus the Eddington limit $L_{E} \approx 10^{47} M_{B H} / 10^{9} M_{\odot} \mathrm{erg} \mathrm{s}^{-1}$ is easily attained; this is indicated by eq. (1), considering that in such events the BHs can double their mass and make $\Delta m \sim M_{B H}$ over short dynamical scales $\Delta t$ matching the effective Eddington time $\eta t_{E} \approx 5 \times 10^{-2}$ Gyr. Given an efficiency for BH formation $M_{B H} / M \sim 10^{-3}-10^{-4}$, the number of bright QSOs tracks the DM clustering, and rises together with the number of host halos exceeding some $10^{10} M_{\odot}$.

Later than $z_{G}$ the accretion feast must cease and the QSO rise turns over. From hierarchical clustering one expects major merging events to become rare at galactic scales, and the prevailing dynamical events to be best described as interactions of mature galaxies within the groups then forming.

In point of fact, connections of QSOs with groups are apparent at $z<1$ by direct imaging of companions or by the statistical evidence of a group-like environment for QSOs and AGNs (see Rafanelli et al. 1995, Fisher et al. 1997). Moreover, a growing number of QSO hosts are found to exhibit the morphological marks of ongoing interactions in spite of their low surface brightness and short duration around 0.5 Gyr, see Hutchings \& Neff 1992, Bahcall et al. 1997, Hasinger et al. 1997, Boyce et al. 1998, McLure et al. 1999). That such interactions occur preferentially in groups is shown by many simulations (see Governato et al. 1996, Athanassoula 2000). This is also expected on the basis of the rate $\tau_{r} \sim 1 / N_{g} \Sigma V$, given the geometrical cross section $\Sigma \sim 4 \pi r_{g}^{2}$, because there the galaxy density $N_{g}$ is high, and the velocity dispersion $V$ is still comparable to the internal galaxy velocities $v_{9}$ (CV98, Kontorovich 2000).

The role of such interactions in QSO evolution (see CV98, Byrd et al 2000) is to cause again the gas in the host to lose angular momentum; so they trigger supply-limited accretion episodes that intermittently rekindle the central BHs. In fact, grazing interactions are shown by simulations to drive up to $50 \%$ of the gas into the central $10^{2}$ pc (see Barnes \& Hernquist 1998). But most episodes originate from a fly-by of the host with bulge mass $M_{o}$ by a companion with mass $M^{\prime}$, at impact parameters $b \gtrsim r_{g}$, over a time scale $\tau \sim 2 r_{g} / V \sim 10^{-1} \mathrm{Gyr}$, comparable to galactic dynamical times. A fractional gas mass sent toward the nucleus $\Delta m / m \sim 5 \%$ is enough to yield $10^{46} \mathrm{erg} \mathrm{s}^{-1}$ in a host containing some $3 \times 10^{9} M_{\odot}$ of gas.

The actual output scales after eq. (1) as $L \propto m(t) \Delta m / m \tau$; over times of order $\tau$ the controlling factor $|\Delta j / j| \gtrsim \Delta m / m$ is statistically dispersed. Many interactions can produce $|\Delta j| j \mid \sim 5 \%$, so the probability of luminosities up to $L \sim 10^{46} \mathrm{ergs}^{-1}$ is $N(L) L \sim$ const. But higher luminosities require closer collisions with a larger companion, and these are fewer; their probability decreases steeply, down to $N L \propto L^{-2}$ when $|\Delta j / j| \approx j M^{\prime} / V b M_{o}$ applies. In sum, the QSO luminosity function $N(L)$ fed by interactions is expected in the form of a double power-law steepening from about $L^{-1}$ to $L^{-3}$. 
Over longer time scales this evolves in two ways. The interactions become rarer as richer groups come of age; e.g., in the absence of strong galaxy evolution, the scaling $\tau_{r}=\tau_{r o}(1+z)^{-1.5}$ results in the critical universe, considering the proportionalities to $1 / N_{g}(z) \propto(1+z)^{-3}$ and to $1 / V \propto M^{-1 / 3}(1+z)^{-0.5}$. This clearly causes a corresponding, weak "density evolution" in the population of bright QSOs. But the interactions also become less effective as $L \propto m(z)$ is fed from host gas reservoirs depleted by all previous discharges. In fact, the gas funneled inward may end up not only in accretion onto the central BH, but also in a less constrained nuclear starburst; such stellar competition raises the overall fraction removed to some $15 \%$ when $5 \%$ is actually accreted.

So the gas is depleted following $d m / d t \approx-0.15 m / \tau_{r}(t)$, to yield $m(t) \propto$ $t^{-0.15 t_{o} / \tau_{\text {ro }}}$. The value of $\tau_{\text {ro }}$ may be derived from the classic local census of bright galaxies with persisting signatures of interactions (Toomre 1977), leading to a time between interactions around $2 \times 10^{2} \mathrm{Gyr}$; this scales down to $\tau_{r o} \approx 1$ Gyr in virialized groups where a density contrast $\approx 2 \times 10^{2}$ is indicated by the hierarchical clustering. The resulting behavior is a strong "luminosity evolution", close to $L \propto t^{-2} \propto(1+z)^{3}$ in the critical universe.

In sum, the QSO luminosity functions driven by interactions will follow

$$
N(L, z) \propto(1+z)^{1.5} L^{-1} \times\left(L / L_{b}\right)^{-2},
$$

with the last factor applying beyond a break at $L_{b} \propto(1+z)^{3}$. In fig. 1 the full results from a systematic numerical computation are represented and compared with the data. The natural normalization is a few \% QSOs per bright galaxy at $z_{G} \approx 2.5$, resulting from the visibility factor $\tau / \tau_{r} \sim 10^{-1}$, and from the number of interaction sites provided by the triple SGs. These comprise up to $40 \%$ of the bright galaxies and include typically one $L_{*}$ galaxy (Ramella et al. 1999).
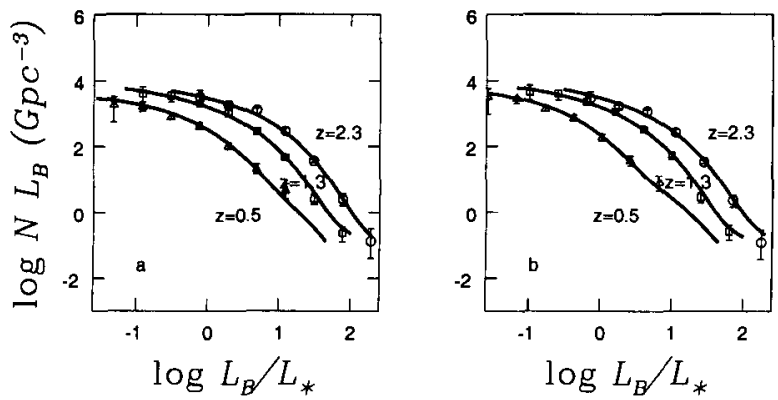

Fig. 1 - Optical luminosity functions of the QSOs powered by interactions of the hosts in galaxy groups. Efficiency $\eta=10^{-1}$, bolometric correction $\kappa_{B}=10, L_{*}=10^{45} \mathrm{erg} \mathrm{s}^{-1}$. Left: Critical cosmology with $\mathrm{h}=0.5$, tilted CDM perturbation spectrum. Right: $\Omega_{0}=0.3, \lambda_{0}=$ $0.7, h=0.65$, CDM perturbations. From Cavaliere \& Vittorini, in preparation.

The thrust of this outline is to show how close to the data comes a simple story of the QSO population based on SGs and richer, later groups as sites of interactions feeding BHs; no free, adjustable $z$-functions are used, and natural, independent values are taken up for the parameters. It is shown by CV98 that similarly good fits obtain in a flat universe with $\Omega_{o}=0.3$ and $h=0.7$. 
A global check on this story is that the average emissions from QSOs or AGNs are expected to go progressively sub-Eddington downward of $z_{G}$, even with constant $\eta \sim 10^{-1}$. The specific prediction is

$$
L / L_{E} \sim[(1+z) / 3.5]^{3} M_{B H}(2.5) / M_{B H}(z) .
$$

The first factor is just the above luminosity evolution due to the depletion of gas available to be accreted upon host interactions; the second (see CV98), is the related mass increase, up to $M_{B H}(0) / M_{B H}(2.5) \sim 5$. The result accords with the trend in the preliminary data, see Haiman \& Menou 1998, Salucci et al. 1998. Another prediction envisages a similar evolution shared by starburst light or reprocessed FIR emission, that compete with $\mathrm{BH}$ accretion for the gas released by such interactions; a point developed by Vittorini et al. 1999.

\section{Small groups and the intracluster medium}

At the other extreme, I shall discuss how the conditions in SGs affect the hot atmospheres of gas that pervade groups and clusters, out to a few Mpcs in the latter case. The central densities are up to $n \sim 10^{-3} \mathrm{~cm}^{-3}$; the temperatures $k T \sim 0.5-10 \mathrm{keV}$ are always close to the virial values $k T_{v}$, making a strong case for dominant gravitational heating of the gas as it falls into the forming DM gravitational wells. By thermal bremsstrahlung the gas emits X-rays at energies $h \nu \sim k T$ with luminosities $L_{X} \sim 10^{42}-5 \times 10^{45} \mathrm{erg} \mathrm{s}^{-1}$.

The scaling of $L_{X} \propto n^{2} R_{X}^{3} T^{0.5}$ after the pure hierarchical, scale-invariant manner - not only $T \approx T_{v} \propto(1+z) M^{2 / 3}$ but also $n \propto(1+z)^{3}$ - would give the relation $L_{X} \propto T^{2}$. Instead the observations provide a shape that steepens with decreasing $T$ toward $L_{X} \propto T^{3}$ for clusters around $k T \approx 4 \mathrm{keV}$, and steepens yet for groups below $1 \mathrm{keV}$; in sum, lower $L_{X}$ at given $T$ (see Ponman 2000).

So the gas apparently resists being piled up in shallow DM wells, and adjusts its internal density $n(T)$ to values decreasing with $T$. The control is conceivably provided by the preheating of the gas with the energy fed back by the stars forming in the member galaxies; specifically, by SN explosions with some addition from stellar winds. The global energy output from forming the stellar mass $\Delta m_{*}$ is canonically evaluated at $E_{*} \approx\left(4 \times 10^{-3} \Delta m_{*}\right) \times 10^{51} \mathrm{erg}$.

The dynamical transfer of momentum by SN explosions and stellar winds to the surrounding gas can directly expel beyond the virial radius some gas mass $\Delta m_{h}$. The proper parameter will be the fractional energy converted into bulk kinetic energy at the escape velocity, that is

$$
\epsilon_{o}=\Delta m_{h} v^{2} / E_{*} \sim 10^{-1},
$$

especially important in the wells with circular $v$ of order a few $10^{2} \mathrm{~km} \mathrm{~s}^{-1}$ that prevail at around $z_{G}$.

The thermal energy fed to the same gas mass $\Delta m_{h}$ results in a temperature

$$
k T_{*} \approx 0.7\left(1-\epsilon_{o}\right) \Delta m_{*} / \Delta m_{h} \gtrsim 0.2 \mathrm{keV} .
$$

But $k T_{v} \approx 0.2 \mathrm{keV}$ is close to the virial value for $M \sim 10^{13} M_{\odot}$; so the SGs constitute a threshold below which stellar preheating can prevent high gas densities 
within the wells. Note that heating the gas amount $\Delta m_{h}$ while expelling it to low densities is a process best suited to generate the rather high "entropy floor" observed in groups, equivalent to $k T_{*} / n^{2 / 3} \sim 100 \mathrm{keV} \mathrm{cm}{ }^{2}$ (Ponman 2000).

Expulsion and heating affect the fraction $f$ of hot gas inside the wells, and its density $n_{i} / n_{e}$ relative to the outside, which appear in the full scaling (apart from a slowly varying shape factor)

$$
L_{X} \propto(1+z)^{3 / 2} f^{2}\left[n_{i} / n_{e}\right]^{2} T^{2} .
$$

Shocks and adiabatic compressions contribute to $n_{i}$ as the gas falls and settles into the wells; I focus on shocks, which explain together the $L_{X}-T$ correlations and the further rise outwards of the entropy in clusters (Ponman 2000).

During the hierarchical growth of a halo with mass $M$, accretion shocks (CMT97) are induced at around the virial radius when the gas falls supersonically. Initially the gas is associated with various merging partners with masses $M^{\prime}$; its temperature $T^{\prime}$ is at the virial or the preheating value $T_{*}^{\prime}$, whichever is larger in each $M^{\prime}$. The ratio $n_{i} / n_{e}$ is set by the jump condition across the shock; depending on $T_{v} / T^{\prime}$ which marks the shock strength, it ranges from 1 to 4 (see CMT97). Correspondingly, $L_{X}$ ranges from 1 to 16 , with some variance.

The upper values obtain in clusters where $T_{v}$ exceeds any external $T_{*}^{\prime}$, the shocks are strong and $L_{X}$ saturates toward $L_{X} \propto T^{2}$. The lower values apply to groups, where the preheating provides $T_{*}^{\prime}$ comparable with the virial $T_{v}$ and the shocks are weak at best; the result is a steeper correlation. Thus the shock model predicts an intrinsically bent $L_{X}-T$ correlation as observed. In parallel, it predicts an entropy deposition $\Delta S \propto \ln T n_{e}^{2 / 3} / T^{\prime} n_{i}^{2 / 3}$ rising outwads.

Meanwhile some adiabatic compression takes place in the settling of the shocked gas into the wells. Compressions and shocks occur over and over again during the hierarchical growth; their balance may be expressed with a polytropic equilibrium, implying the radial distribution $T(r) \propto n^{\Gamma-1}(r)$ with $1<\Gamma<1.3$ (see Cavaliere \& Fusco-Femiano 1978, Tozzi \& Norman 1999). This converts, as it were, the previous history of shocks and compressions in the cluster into a space stratification. The derived $L_{X}-T$ relation, including the shape factor from the profiles of $n(r)$ and $T(r)$, constitutes the intermediate step to compute the X-ray luminosity functions and the source counts using standard formulae.

Two complications arise. The expression for $L_{X}$ is to be convolved over all "merging histories" leading to $M$, that is, over various sequences of accreted clumps $M^{\prime}$ with their probabilities. In such average one has to include the effects of the dynamical and of the thermal feedback, namely: the gas fraction $\Delta m_{h} / m_{h}$ expelled from clumps $M^{\prime}$ and heated to $T_{*}^{\prime}$ by the internal SN activity; the complementary fraction still remaining inside $M^{\prime}$ at the virial $T_{v}^{\prime}$. During the merging events both components fall into the main halo $M$, are shocked with different strengths, and contribute to $L_{X}$ in proportion (see MC99).

All that sounds complex, but in fact it joins nicely with the "semi-analytic models" (SAMs) developed by several groups (Munich: Kauffmann et al. 1993; Durham: Cole et al. 1994; Santa Cruz: Somerville \& Primack 1998) to treat the processes governing the star and galaxy formation, and to fit the optical luminosity functions. The SAMs are based on the same hierarchical merging histories said above. They envisage the baryons to condense into quiescent star formation at the minima of the forming DM potential wells, upon cooling down 
from a diffuse hot phase at the virial temperature. Such implied hot component constitutes just the intracluster or intragroup gas emitting in X-rays.

In fact, the treatment of the hot gas as outlined above can be grafted (MC99) onto the SAMs, because it proceeds from the same basic equations and uses the same parameters. The main one tunes the feedback per unit stellar mass $\Delta m_{h} / \Delta m_{*}=\left(150 \mathrm{~km} \mathrm{~s}^{-1} / v\right)^{\alpha_{h}} ;$ this affects indirectly the star formation rate since it offsets the cooling, and directly $L_{X}$ as shown by eqs. (4) - (6). Values of $\alpha_{h} \approx 5$ as in the original SAM from Durham correspond to thorough expulsion of baryons from the shallow wells that prevail at high $z$. This leads (see fig. 2) to a star formation rate declining considerably from $z \approx 1.5$ to 5 , but also to low counts of the X-ray sources associated with the hot intragroup medium. More "neutral" values $\alpha_{h} \lesssim 2$, corresponding to $\epsilon_{o} \propto v^{2-\alpha_{h}} \approx$ const, yield (see fig. 2) high star formation out to $z \approx 4$, together with soft X-ray counts considerably enhanced at fluxes $F_{X} \lesssim^{-15} \mathrm{erg} \mathrm{cm}^{-2} \mathrm{~s}^{-1}$, within the reach of Chandra and of XMM.
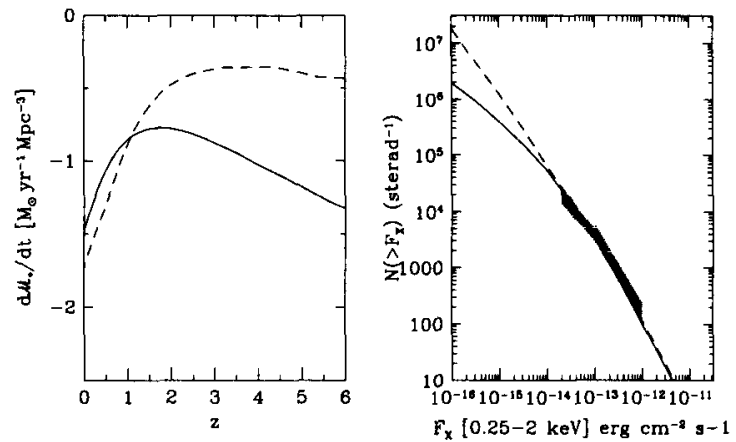

Fig. 2 - Left: The star formation rate, observable in the optical, from baryon processing during the hierarchical clustering. Solid line: the peaked shape from the original Durham parameters, primarily $\alpha_{h}=5.5$, that enhance the feedback in shallow wells; dashed line: the shape flat toward high $z$ from the "neutral" set including $\alpha_{h}=1.5$. Right: The corresponding source counts $N\left(>F_{X}\right)$ in the X-ray band $0.5-2 \mathrm{keV}$. Critical universe, $h=0.5$. See MC99.

These results are to be seen in the light of the current debate concerning the intrinsic star formation rate at high $z$ (see Ellis 1998) as derived from the $\mathrm{O}-\mathrm{IR}$ emissions of the stellar baryons in early galaxies. The $\mathrm{X}$-rays from the surrounding hot baryons will concur or compete with these data to gauge directly the feedback process crucial to star and galaxy formation.

\section{Conclusions}

SGs play a pivotal role in breaking at the level of baryonic processes the gravitational scale-invariance of the hierarchical formation of cosmic structures.

Concerning the QSOs, the break is due to the transition: strong merging - interactions as dominant drivers for the evolution of the host galaxies. The result is a limit to, and the eventual depletion of, the cold gas available in the 
hosts for accretion onto the central BHs. This in turn causes the early QSO rise to turn into a steep fall later than $z \approx z_{G} \approx 2.5$.

Concerning the X-ray sources from the hot atmospheres pervading the DM wells, the scale-invariance is broken when the stellar feedback yields to gravitational energy liberation by the infalling gas. This occurs in groups larger than SGs, with stronger feedback allowing less baryons and weaker emissions.

Thus both kinds of sources signal the significance of SGs out to $z \approx 2.5$.

\section{References}

Athanassoula, E. 2000, these proceedings

Bahcall, J. N., Kirhakos, S., Saxe, D. H. \& Schneider, D. P. 1997, ApJ, 479, 642 Barnes, J. E. \& Hernquist, L. E. 1998, ApJ, 495, 187

Boyce, P. J. et al. 1998, MNRAS, 298, 121

Boyle, B. J., Shanks, T. \& Peterson, B.A. 1988, MNRAS, 235, 935

Byrd, G. G., Valtonen, M. J., 2000, these proceedings

Cavaliere, A, \& Fusco Femiano, R. 1978, A\&A, 70, 767

Cavaliere, A., Menci, N., Tozzi, P., 1997, ApJL, 484, 1 (CMT97)

Cavaliere, A., \& Vittorini, V. 1998, ASP CS, 146, 26 (CV98)

Cole, S., Aragon-Salamanca, A., Frenk, C. S., Navarro, J. F., \& Zepf, S. E. 1994, MNRAS, 271, 781

Ellis, R. 1998, Nature, 395, 3

Fisher, K. B., Bahcall, J. N., Kirhakos, S. \& Schneider, D. P. 1996, ApJ, 468, 469

Governato, F., Tozzi, P. \& Cavaliere, A. 1996, ApJ, 458, 18

Haehnelt, M. G., Natarajan, P. \& Rees, M. J. 1998, MNRAS, 300, 817

Haiman \& Menou 1998, preprint [astro-ph/9810426]

Hasinger, G. et al. 1997, preprint "Interacting Galaxies, the X-ray View"

Hutchings, J. B. \& Neff, S. G. 1992, AJ, 104, 1

Kauffmann, G., White, S. D. M., \& Guiderdoni, B. 1993, MNRAS, 264, 201

Karachentsev, I. D., Byrd, G.'G., Chernin, A. D. \& Valtonen, M.J. 1999, Fund. Cosmic Phys., in press

Kontorovich, V. M. 2000, these proceedings

McLure et al. 1998, preprint [astro-ph/9809030]

Menci, N. \& Cavaliere. A. 1999, preprint [astro-ph/9909259] (MC99)

Osmer, P. S. 1998, ASP CS, 146, 1

Ponman, T. J., Lloyd-Davies, E., Helsdon, S., 2000, these proceedings

Rafanelli, P., Violato, M. \& Baruffolo, A. 1995, AJ, 109, 1546

Ramella, M., et al. 1999, A\&A 342, 1

Rees, M. J. 1984, ARAA 22, 471

Salucci, P., Szuszkievicz, E., Monaco, P. \& Danese, L. 1998, preprint [astro$\mathrm{ph} / 9811102]$

Shaver, P. A. et al. 1996, Nature, 384, 439 
Somerville, R. S., \& Primack, J.R. 1998, preprint [astro-ph/9802269]

Ste6idel, C. C., Adelberger, K. L., Giavalisco, M., Dickinson, M. E. \& Pettini, M. 1999, ApJ, 519, 1

Toomre, A. 1977, in Evolution of Galaxies and Stellar Populations, ed. B.M. Tinsley \& R.B. Larson (Yale Univ. Obs.: New Haven) p. 401

Tozzi, P. \& Norman, C. 1999, preprint [astro-ph/9905046]

Vittorini, V, Malquori, D. \& Guiderdoni, B. 1999, in preparation 